\title{
Die Alchemie im Spiegel der schönen Literatur*
}

\author{
Von Johanna Bleker
}

\section{Zum Ziel der Untersuchung und zur Auswahl der Literatur}

Bei allem Bemühen um die Geschichte der Alchemie fällt es uns bisweilen schwer zu begreifen, daß die Menschen jahrhundertelang an diese Kunst geglaubt haben. Wir gehen davon aus, daß die Transmutation der Metalle nach den Regeln der Alchemie unmöglich war, und wir fragen uns, warum die Alchemisten, deren Versuche doch fehlschlagen mußten, dies nicht ebenfalls eingesehen haben. Die geistige Welt der damaligen Menschen und ihre Haltung zur Alchemie ist uns heute weitgehend fremd geworden.

Aus diesem Grund ist es förderlich, nicht nur die alchemistische Fachliteratur, sondern auch die Darstellungen der schönen Literatur zu betrachten. Denn das Bild der Alchemie in der schönen Literatur dürfte - wenigstens zu gewissen Zeiten - die allgemeine Auffassung über diese Kunst wiederspiegeln.

Alchemie und Literatur können auf verschiedene Weise miteinander verbunden sein. Einige Formen möchte ich gleich zu Beginn abgrenzen.

So muß bei dieser Untersuchung das alchemistische Lehrgedicht außer acht gelassen werden. Denn diese Lehrdichtung ist nicht Alchemie in der Literatur, sondern Literatur der Alchemie. Und wenn ich hier versuchen möchte, durch die Aussagen der zeitgenössischen Literaten sozusagen eine Außenansicht der Alchemie zu finden, so kann die Literatur der Alchemie selbst kaum etwas dazu beitragen.

Auch eine Untersuchung über die Verwendung alchemistischer Begriffe in der Dichtkunst ist für unsere Frage nicht sehr aufschlußreich. Ich möchte dies an einigen kurzen Beispielen aus dem dichterischen Werk des Engländers John Donne zeigen. John Donne lebte von 1572-1631. In seinen äußerst bildreichen Gedichten verwendet er auch zahlreiche alchemistische Allegorien ${ }^{1}$.

* Vortrag, gehalten auf dem 3. Nordwestdeutschen Kolloquium für Wissenschaftsgeschichte in Kiel, am 3. Juli 1971.

1 Vgl. Edgar Hill Duncan, Donne's Alchemical Figures, A Journal of English Literary History E.L.H. (Baltimore) 9 (1942) 257-285, und Joseph Anthony Mazzeo, Notes on John Donne's Alchemical Imagery, in Renaissance and Seventeenth-Century Studies, London/New York 1964, S.60-89. 
In seinem Gedicht «Love's Alchemy» vergleicht er das Glück der Liebe mit dem Stein der Weisen: Das Glück der Liebe sei ebensowenig zu finden, wie das Elixier. Und ebenso wie der Alchemist sein Destilliergerät preise, wenn bei seiner Suche wenigstens eine Arznei oder eine wohlriechende Substanz abfiele, so gehe es auch dem Liebenden, der von höchstem und langwährendem Entzücken träume und schon froh sei, wenn ihm eine kurze Sommernacht wie eine lange Winternacht erscheine:

„Oh 'tis imposture all:

And as no chymique yet th'Elixar got,

But glorifies his pregnant pot,

If by the way to him befall

Some odoriferous thing, or medicinall,

So, lovers dreame a rich and long delight

But get a winter-seeming summer's night." ${ }^{2}$

An anderer Stelle, in dem Gedicht «Resurrection (imperfect)», verwendet John Donne den alchemistischen Vergleich des Todes und der Auferstehung Christi mit der Gewinnung der Tinktur aus dem natürlichen Gold: Der Auferstandene, als die Tinktur, hat die Macht, bleierne und eiserne Herzen zum Guten zu wandeln und das sündige Fleisch dem seinen gleich zu machen:

„Hee was all gold when he lay downe, but rose

All tincture and doth not alone dispose

Leaden and iron wills to good but is

Of power to make even sinfull flesh like his." 3

Diese zwei kurzen Beispiele zeigen, daß es für unsere Untersuchung nicht sehr ergiebig ist, der Verwendung alchemistischer Motive in der Dichtkunst weiter nachzugehen. Die dichterische Sprache verwendet die Bilder je nach der gewünschten Aussage im positiven wie im negativen Sinn. So können wir Donnes Gedichten nur entnehmen, daß ihm die Gedankenwelt der Alchemie sehr vertraut gewesen sein muß. Eine bestimmte Ansicht über die Alchemie kann jedoch aus diesen Gedichten nicht abgeleitet werden.

2 John Donne, John Donne, Dean of St.Paul's, Complete Poetry and Selected Prose, Herausgeber: John Hayward, 10. Auflage, London 1967, S. 28.

3 Ebd. S. 290. 
Deshalb muß sich diese Untersuchung auf die schöne Literatur beschränken, die die Alchemie und den Alchemisten direkt zu ihrem Gegenstand gewählt hat. Dabei sind freilich nur die Werke von Interesse, die zu einer Zeit entstanden sind, in der die Königliche Kunst noch blühte und die Gedanken der Zeitgenossen bewegte. Denn nur die Autoren dieser Zeit können uns unmittelbar zeigen, wie die Alchemie damals gesehen und begriffen wurde.

Die Literatur, die ich ausgewählt habe, entstammt dem Mittelalter und der frühen Neuzeit. Es handelt sich dabei nur um Beispiele aus den bedeutendsten dichterischen Werken der damaligen Zeit und nicht um den Versuch, alle dichterische Aussage, die über die Alchemie gemacht wurde, zu erfassen ${ }^{4}$.

Das früheste Werk, das ich erwähnen möchte, ist der von JEAN DE MEUN verfaßte zweite Teil des Roman de la Rose. Er entstand am Ende des 13. Jahrhunderts. Das späteste Werk, auf das ich eingehen möchte, ist Ben Johnsons Schauspiel The Alchemist, das im Jahre 1610 uraufgeführt wurde.

In der dazwischen liegenden Zeitspanne habe ich bei Dante, Petrarca Sebastian und Brandt eine Erwähnung der Alchemie gefunden. Die ausführlichste Darstellung ist jedoch in den Canterbury Tales enthalten, die Geoffrey Chaucer gegen Ende des 14. Jahrhunderts verfaßte.

Im folgenden soll untersucht werden, wie in diesen Werken die Alchemie verstanden wird und mit welchen Argumenten die Auseinandersetzung um ihre Möglichkeiten geführt wird.

\section{Die Bewertung der Alchemie im «Roman de la Rose»}

Ich beginne mit dem Roman de la Rose, als der vermutlich ältesten belletristischen Darstellung der Alchemie (die von mir benutzte Textfassung stammt allerdings aus dem frühen 16. Jahrhundert).

Der erste Teil des Rosenromans, der zwischen 1220 und 1240 entstand, ist eine allegorische Minnedichtung. Der zweite Teil wurde um 1280 von

4 Als Übersichten über die Behandlung der Alchemie in der Dichtung vgl. Friedrich Gustav Hartlaub, Von der Alchemie in der Dichtung, Basf 2 (1952) 127-135; JohN Read, The Alchemist in Life, Literature, and Art, London 1947; JoHn ReaD, Alchemy and Literature, Proc.Chem.Soc. (London) 1957 138-143; Kurt Seligmann, Das Weltreich der Magie, 5000 Jahre Geheime Kunst, Stuttgart 1958, S. 136-142. 
Jean de Meun verfaßt und ist ein lehrreich interessantes Zeitgemälde im allegorischen Gewand ${ }^{5}$.

Für Jean de Meun ist die Alchemie eine wirkliche Kunst:

«Non pourtant, c'est chose notable

D'arquemie est art véritable,

Qui saigement en ouvreroit

Grans merveilles y trouveroit ... ${ }^{6}$

Indessen ist sie wie jede Kunst unvollkommen. Denn obwohl die Kunst, die die Natur nachahmen und ihr so bei ihrem Werke helfen soll, aufmerksam vor der Natur auf den Knien liegt, gelingt es ihr nur, wie ein Affe, das Vorgemachte nachzumachen. Während die Natur mühelos die Stoffarten ineinander umwandelt, gelingt dies der Kunst nur unter großen und langwierigen Anstrengungen. Die Transmutation ist jedoch keineswegs unmöglich. So wie die Natur Blitz und Donner in Steine verwandelt und zur Erde schleudert, so verwandelt die Alchemie Farnkraut in Glas ${ }^{7}$. - (Das Wort Fougière $=$ Farnkraut hat die Doppelbedeutung Glas. Hier ist vermutlich die Verwendung von Pflanzen-Pottasche zur Glasherstellung gemeint.) - Der Weise erkennt - so erklärt DE MEun - daß es sich in beiden Fällen um transmutierte Arten handelt, deren Teile in der Substanz und in der Form verändert sind. Einmal durch die Kunst, einmal durch die Natur:

«Si sont les especes changées

Et leurs pieces d'eulx estrangées

et en substance et en figures

Ungs par Art, autres par Natures... ${ }^{8}$

In aufmerksamer Nachfolge der Natur ist auch die Transmutation der Metalle möglich ${ }^{9}$. Allein jene, die es mit sophistischen Spitzfindigkeiten versuchen, werden es der Natur niemals gleichtun können:

5 Zu Jean de Meun vgl. auch Frederic Walker, Jean de Meun and Alchemy, Journal of Chemical Education 7 (1930) 22 863-22 874.

6 JeAn de Meun, Le Roman de la Rose. Dans la version attribuée à Clément Marot, Herausgeber S.F.BARIDon, Milano/Varese 1957, Zeile 16460-16463.

7 Vgl. ebd. Zeile 16442-16482.

8 Ebd. Zeile 1648616489.

9 Vgl. ebd. Zeile $16490 \mathrm{ff}$. 
«Mais iceulx or ne feront mie

Qui ouvrent de sophisterie

Travaillent tant comme ilz vourront

Ja nature n'aconsuyvront... ${ }^{10}$

Es wird sich zeigen, daß Jean de Meun der einzige Autor ist, der die Alchemie so rückhaltlos bejaht. Vor allem die praktische Durchführbarkeit wird in der späteren Literatur vielfach bestritten.

Die Verurteilung der Alchemie bei Dante und Petrarca

Schon zu Beginn des 14. Jahrhunderts werden die ersten Anfänge einer Kritik sichtbar.

Dante, der Dichter der Göttlichen Komödie, beschreibt die Leiden von zwei Alchemisten, die in den untersten Kreis der Hölle verbannt worden sind, in die Hölle der Fälscher ${ }^{11}$. Einer der Verdammten erklärt: «Ich bin der Schatten des Capocchio, der mit der Alchemie die Metalle fälschte; und du erinnerst dich ... daß ich ein guter Affe der Natur war.» ${ }^{12}$

Diese Redewendung zeigt die Frechheit des Betrügers. Die Alchemie soll ein Affe der Natur sein, indem sie ihre Werke getreulich nachbildet.

Capocchio aber äffte die Natur durch die Herstellung von falschem Gold. Die Verdammung der Alchimisten in die tiefste Hölle verurteilt nicht die Alchemie, sondern den mit dieser Kunst betriebenen Mißbrauch.

Diese Verurteilung entspricht dem Alchemieverbot durch Papst Johannes XXII. Seine Bulle «Spondant quas non exhibent ...» wurde 1317, vier Jahre vor Dantes Tod, erlassen. Sie richtet sich gegen den Betrug und gegen die Irreführung der Armen durch die Alchemisten, also nicht gegen die Alchemie selbst ${ }^{14}$.

10 Ebd. Zeile 16522-16525.

11 Zu Dante vgl. auch Edmund O. v. Lippmann, Chemisches und Technologisches bei Dante, Archivio di Storia della Scienza (Roma) 3 (1922) 45-56.

12 Dante Alighieri, Cantica del divino poeta Danthe Alighieri Fiorentino, Venedig 1536, 29. Gesang

Fol. 173 v «Sivedrai, ch'i son l'ombra di Capocchio

Che falsai li matalli con alchimia:

Et ten' dee ricordar, se ben t'adocchio,

Com' i fu di natura buona scimia.»

13 Vgl. Wilhelm Ganzenmüller, Die Alchemie im Mittelalter, Paderborn 1938 (Nachdruck Hildesheim 1967) S. 84 f. und HermanN KopP, Die Alchemie in älterer und neuerer Zeit, Band I, Heidelberg 1886 (Nachdruck Hildesheim 1962) S. 225. 
Die gleiche Haltung liegt auch den Angriffen zugrunde, die etwa 40 Jahre später von Petrarca formuliert werden. Sein um 1360 entstandenes Buch De remediis utriusque fortunae enthält ein Kapitel «De Alchemia», in dem Vernunft und Hoffnung über die Möglichkeit streiten, durch die Alchemie reich zu werden. Mit dem Hinweis auf die Erfahrung, daß alle Versprechungen der Alchemisten nichts als Betrug seien, verurteilte die Vernunft die ganze Kunst: Trug und Schande seien die einzigen Ergebnisse der Alchemie. Noch nie habe man gesehen, daß durch diese Kunst ein Armer reich geworden sei. Wohl aber sei schon manch ein Reicher durch sie arm geworden. Doch Geldgier und Leichtgläubigkeit trüben den Verstand der Menschen derart, daß sie gegen ihre Erfahrung an das glauben, was sie begehren ${ }^{14}$.

Die Ratio schildert die schrecklichen Folgen der alchemistischen Bemühungen in den buntesten Farben:

«Dein Haus füllt sich mit absonderlichen Gästen und wunderlichen Vorrichtungen, als da sind Fresser und Säufer - sehr natürlich, trocknet doch das Feuer und die Gier die Leiber aus -, Ohrenbläser Windbeutel und Spötter, als da sind Ascheneimer Tiegel, Phiolen voll stinkender Wässer, ausländische Kräuter, Salze aus fernen Ländern, Schwefelbrocken, Destilliergefäße und Öfen; aus alle dem entspringt dir fruchtlose Sorge, Verdumpfung des Gemütes, Entstellung des Angesichtes, stierer Blick - und schließlich naht die Armut, die du herausgefordert hast, ja, was schlimmer ist, dein Ruf wird der eines Gauners und dein Leben das eines Diebes, zugebracht in der nächtlichen Verborgenheit übelberüchtigter Schlupfwinkel.» ${ }^{15}$

Petrarca nennt Geldgier und Dummheit als die eigentlichen Motive für den Glauben an die Alchemie. Seine Ablehnung stellt sich auf die Grundlage der Vernunft und der praktischen Erfahrung. Sie trifft aber ebenfalls nur den Mißbrauch und nicht den eigentlichen Kern der Alchemie.

14 Vgl. Francesco Petrarca, De remediis utriusque fortunae, Libri II, Leyden 1572, Buch I, Kap. 111, S.381-383.

15 Zitiert in der Übersetzung von Lippmann, aus Edmund O. v. LiPpManN, Petrarca über die Alchemie, Archiv für Geschichte der Naturwissenschaften und der Technik 6 (1913) 236-240. 


\section{Die Darstellung der Alchemie in Chaucers «Canon's Yeoman's Tale»}

Eine Diskussion aller Aspekte der Alchemie finden wir dagegen bei Geoffrey Chaucer. In seinem «Canon's Yeoman's Tale» ${ }^{16}$ - der Geschichte vom Dienstmann des Kanonikus - entwirft er zuerst das Bild des ernsthaft arbeitenden, gläubigen Alchemisten, enthüllt dann die Kunststücke der alchemistischen Scharlatane und diskutiert schließlich die grundsätzlichen Möglichkeiten der Alchemie. Es lohnt sich, diese Geschichte etwas eingehender zu betrachten ${ }^{17}$.

Geoffrey Chaucer lebte vermutlich von 1340 bis 1400. Er war Beamter am anglischen Königshof und zugleich ein Mann, der über vielfältige literarische und wissenschaftliche Kenntnis verfügte. Man nimmt an, daß sein berühmtestes Werk, die Canterbury Tales, im Jahre 1386 begonnen wurde. "The Canon's Yeoman's Tale» gilt als eine der schönsten und originellsten Geschichten unter den Canterbury Tales ${ }^{18}$.

Der Beginn der Geschichte ist dramatisch: Der Kanonikus und sein Dienstmann erreichen die Gesellschaft, die nach Canterbury zieht, in einem recht aufsehenerregenden Zustand. Ihre Reittiere sind abgehetzt und erschöpft, ihre Kleidung ist abgerissen und zerlumpt. Der Verdacht liegt nahe, daß die beiden sich auf der Flucht befinden. Voll Neugier fordert der Leiter der Reisegesellschaft den Dienstmann auf, über ihr Schicksal zu berichten. Der Kanonikus sucht seinen Diener daran zu hindern, das zu erzählen, was geheim bleiben müsse. Als ihm dies nicht gelingt, flieht er in Kummer und Beschämung.

Nun sind der Erzählfreude des Dieners keine Grenzen mehr gesetzt. Der ungelehrte Mann, der sieben Jahre lang an den Blasebälgen und Retorten seines von der Alchemie besessenen Herrn gearbeitet hat, entwirft ein detailliertes Bild der Alchemie seiner Zeit.

16 Geoffrey Chaucer, The Canon's Yeoman's Tale, in A Six-Text Print of Chaucer's Canterbury Tales in Parallel Colums. (from the ms : 1. The Ellesmere, 2. The Hengwrt 154, 3. The Cambridge Univ. Libr. Gg. 4.27, 4. The Corpus Christi College, Oxford, 5. The Petworth, 6. The Lansdowne 851, British Museum), Herausgeber Frederick J. Furnivale, London 1870 (?), (Nachdruck New York 1967). Part VII, Fol. 547-574. Die wörtlichen Zitate entsprechen der Textfassung des Ellesmere MS.

17 Vgl. John Read, The Alchemist in Life, Literature and Art, London 1947, S. 29 ff. und Frederic Walker, Geoffrey Chaucer and Alchemy, Journal of Chemical Education 9 (1932) 1378-1385.

18 Vgl. Nevill Coghill, Chaucer's Life - Chaucer's Work's, Einleitung zu Geoffrey Chaucer, The Canterbury Tales, translated into Modern English, Herausgeber N. CogHILL. Harmondsworth, Middlesex 1970 (= Penguin Classics). 
Er berichtet von den fruchtlosen alchemistischen Prozessen, die sein Herr ausführte. Er zählt die zahlreichen verwendeten Substanzen auf. Er beschreibt die Ratlosigkeit nach dem Mißlingen der Experimente. Er schildert die Unermüdlichkeit, mit der nach der unvermeidlichen Explosion die Scherben wieder zusammengefegt und die Metallreste gesammelt werden, um es ein weiteres Mal zu versuchen. Erbittert läßt er sich über den Schwefelgestank aus, der den Alchemisten so anhaftete, daß er meilenweit zu erkennen sei. Die Alchemie, das ist die Quintessenz dieses Berichtes, ruiniert jeden, der sich mit ihr abgibt. Sie bringt keine Reichtümer, sondern Schulden und Elend.

Der Dienstmann entwirft hiermit zunächst das Bild der profanen Alchemie, deren Anhänger in England verächtlich «Puffer» - Blasebalgpuster genannt wurden. Im Gegensatz zu diesen vornehmlich experimentierenden Pustern standen die gelehrten Alchemisten, deren erstes Anliegen es war, in die hermetischen Schriften einzudringen und den Stein der Weisen sozusagen auf philosophischem Wege zu ergründen.

Klar wird, daß die Experimentierarbeit in der Alchemistenküche vergeblich bleibt. Immer wieder unterbricht der Dienstmann seine Erzählung, um das zu betonen, zuweilen klagend, zuweilen mit Worten der Erbitterung und des Spotts. Und doch wird die Alchemie niemals grundsätzlich abgelehnt.

Die Warnung, daß alles Bemühen um diese Kunst vergeblich sein müsse, baut nicht auf der Feststellung auf, daß die Transmutation grundsätzlich unmöglich sei. Die Ursache des Mißerfolges liegt vielmehr in der Schwäche des menschlichen Verstandes: Diese Wissenschaft ist unseren Möglichkeiten so weit voraus, daß wir sie niemals einholen können. Sie entgleitet unseren Bemühungen und macht uns alle zu Bettlern:

„But that science, is so fer us beforn

We mowen nat al though we hadden sworn

It ouer-take, it slit awey so faste

It wole us maken beggers in the laste ..."19

Der zweite Teil der Erzählung handelt von den betrügerischen Praktiken der vorgeblichen Alchemisten. Der Dienstmann berichtet ausführlich über die verschiedensten Machenschaften eines solchen Betrügers. Auch dieser ist wiederum ein Kanonikus, «a Chanon of Religioun». Der Dienst-

19 Chaucer, The Canon's Yeoman's Tale, Ellesmere MS, Zeile 680-683. 
mann legt jedoch Wert darauf, daß solche Schwindler von den ehrlichen Alchemisten streng zu unterscheiden sind. Man soll die ehrlichen Alchemisten nicht nach denen beurteilen, die ihre Kunst mißbrauchen, und bedenken, daß sich selbst unter den zwölf Aposteln ein Verräter befand. Sollte man aber wegen des einen Judas alle anderen tadeln?:

„,That among cristes Apostles twelue

Ther was no traytour, but Iudas hym selue

Thanne why sholde all the remenant haue a blame

That giltlees were ..." 20

Zum Schluß gibt der ungebildete Dienstmann des Kanonikus noch seine Meinung über die Schriften der alten Philosophen ab, mit denen sich die gelehrten Alchemisten befassen.

Auch diese Bemühungen um die Alchemie müssen erfolglos sein, denn die Sprache der hermetischen Schriften ist so nebelhaft, daß sie heute kein Mensch mehr verstehen kann.:

„Philosophres speken so mystily

In this craft that man kan nat come thereby..." 21

Die Erklärung dafür ist bei den alten Philosophen selbst zu finden. Die Autorität, auf die der Dienstmann sich beruft, ist «Arnold of the newe towne». Im Rosarium des Arnold von Villanova stehe geschrieben, daß schon der erste Vater der Philosophen, nämlich Hermes, die Anweisung gegeben habe, die Alchemie geheimzuhalten. Nur wer die Absicht und die Sprache der Philosophen verstehe, dürfe diese Kunst ausüben ${ }^{22}$. - (Diese Anweisung zur Geheimhaltung stammt nach Duncan nicht aus dem Rosarium, sondern aus dem Buch De Secretis naturae des Arnold von Villanova, ist aber sonst sinngetreu wiedergegeben) ${ }^{23}$.

Ist es aber überhaupt möglich, die Absicht und die Sprache der Philosophen zu verstehen? Die Antwort hierauf ist im Buch Senior enthalten, das der Dienstmann als nächstes anführt. - (Mit dem Buch Senior ist die Senioris Zadith Tabula gemeint. Nach den Untersuchungen von Duncan

20 Ebd. Zeile 1002-1005.

21 Ebd. Zeile 1394-1395.

${ }^{22}$ Vgl. ebd. Zeile 1428-1447.

23 Vgl. Edgar H. Duncan, The Literature of Alchemy and Chaucer's Canon's Yeoman's Tale: Framework, Theme, and Characters, Speculum 43 (1968) 633-656. 
und Ruska hat Chaucer sich auch hier im wesentlichen an die Vorlage gehalten ${ }^{24}$.

In der von Chaucer umgeänderten Fassung handelt es sich um ein Gespräch, das Plato mit seinem Schüler führt ${ }^{25}$. Plato beantwortet die Frage nach dem Stein der Weisen mit immer anderen Umschreibungen der Sache: Der Stein werde Titanos genannt, dies sei Magnesia, und dies sei ein aus den vier Elementen zusammengesetztes Wasser.

Der Schüler zeigt sich unzufrieden über die Erklärung des Unbekannten mit dem Unbekannteren. Doch Plato weigert sich, eine konkrete Antwort zu geben. Es sei den Philosophen verboten, ihr Wissen anderen Menschen mitzuteilen. Denn das Geheimnis der Alchemie sei Christus so Jieb und wert, daß die Offenbarung dieses Geheimnisses allein seinem göttlichen Willen vorbehalten sei:

„Nay nay quod Plato, certein that I nylle The Philosophres, sworn were euerychoon That they sholden discouere it un-to noon $\mathrm{Ne}$ in no book it write, in no manere ffor un-to Crist, it is so lief and deere That he wol nat that it discouered bee But where it liketh to his deitee..." 26

Hiermit führt Chaucer sein letztes und ernsthaftestes Argument gegen die Alchemie ins Feld. Schon im ersten Teil der Geschichte hatte er gewarnt, daß der Verstand des Menschen zu armselig sei, um diese hohe Wissenschaft zu begreifen. Nun erklärt er diese Unmöglichkeit zur Absicht Gottes. Wie aber kann der Mensch gegen den Willen Gottes etwas erreichen. Er kann sich nur versündigen, wenn er es dennoch versucht:

„Thanne conclude I thus, sith that god of heuene

Ne wil nat that the Philosophres neuene

How that a man, shal come un-to this stoon

I rede us for the beste, lete it goon

ffor who so, maketh God his Aduersarie

As for to werken, any thyng in contrarie

${ }^{24}$ Vgl. Duncan, ebd. und Julius Ruska, Chaucer und das Buch Senior, Anglia, Zeitschrift für englische Philologie 61 (1937) 136-137.

25 Vgl. Chaucer, Ellesmere MS, Zeile 1448-1471.

26 Ebd. Zeile 1463-1469. 
Of his wil, certes neuer shal be thryue Thogh that he multiplie terme of lyue And there a poynt for ended is my tale God sende euery trewe man, boote of his bale."

«Und hier endet meine Geschichte; Gott helfe jedem redlichen Mann in seinen Sorgen. ${ }^{27}$

Es ist eindeutig die Absicht Chaucers mit seinem «Canon's Yeoman's Tale» jedermann vor der Ausübung der Alchemie zu warnen. Mit unvergleichlicher Eindringlichkeit demonstriert er die Erfolglosigkeit aller alchemistischen Bemühungen, und nirgends wird der mit der Alchemie betriebene Betrug ausführlicher geschildert, als in dieser Geschichte.

Und doch ist Chaucer kein ausgesprochener Gegner der Alchemie, wie dies zuweilen von Literaturhistorikern behauptet wird, denen die Vorstellung, der große Dichter sei einem Irrglauben angehangen, Unbehagen zu bereiten scheint ${ }^{28}$. Wenn Elias Ashmole in seinem Theatrum Chemicum Britannicum von 1652 CHAUCER einen Meister der Alchemie nennt, ,, ranked amongst the Hermetic Philosophers", tut er das mit einem gewissen Recht $^{29}$ :

So ist zunächst zu bemerken, daß CHAUcer eine strenge Unterscheidung zwischen dem ehrlichen Alchemisten und dem Betrüger vornimmt und nicht, wie Petrarca, die Alchemie schlechthin zum Betrug erklärt. Man kann auch nicht von einer grundsätzlichen Ablehnung der Alchemie sprechen. Denn Chauger behauptet nicht, daß die Vollendung des Magisteriums prinzipiell unmöglich sei. Seine Zweifel am Gelingen des Großen Werkes entstammen vielmehr einer ganz bestimmten Geisteshaltung des späten christlichen Mittelalters: Die Erkenntnisfähigkeit des Menschen hängt von der Gnade Gottes ab, und so kann nicht das Streben des Menschen, sondern nur Gottes Wille das Geheimnis der Alchemie entdecken.

Gerade diese mittelalterliche Haltung ist es, die Chaucer dazu befähigt, ein wahrhaft treffendes Bild der Alchemie seiner Zeit zu zeichnen. Denn der geistige Hintergrund, dem seine Argumente entstammen, ist der gleiche,

27 Ebd. Zeile 1472-1481.

${ }^{28}$ Vgl. Ben Jonson, The Alchemist, edited with introduction, notes, and glossary by Charles Montgomery Hathaway. New York 1903 (= Yale Studies in English, Ed. A.S.Cook, Nr. 17), Einleitung S. 66.

29 Elias Ashmole, Theatrum Chemicum Britannicum. Containing Severall Poeticall Pieces of our Famous English Philosophers who have written the Hermetique Mysteries in their owne Ancient Language, Teil I, London 1652, S. 470. 
vor dem sich die abendländische Alchemie zu ihrer höchsten Blüte entfaltete.

\section{Sebastian Brandt und Ben Jonson: Die Alchemie als Betrug}

Petrarcas vernunftbetonte Ablehnung des augenscheinlich Unmöglichen entspricht nicht mehr diesem mittelalterlichen Denken, obwohl sie zeitlich früher liegt als Chaucers Geschichte. Petrarcas Kritik deckt sich mit der der späteren Autoren. So finden wir ähnliche Argumente bei Sebastian Brandt, der in seinem 1494 erschienenen Narrenschiff nur kurz vom "großen beschiß der alchemy» handelt ${ }^{30}$. Die betrügerischen Alchemisten befinden sich in Gesellschaft derer, die «den tumen ... zu dem fleysch $»^{31}$ wiegen und «Müsdreck ... under pfeffer» ${ }^{32}$ mischen. Nur ganz nebenher bemerkt er, daß die Alchemie schlechthin unmöglich sei:

«Dann Aristoteles der gycht

Die gstalt der ding wandeln sich nicht. ${ }^{33}$

Diese kurze Bemerkung ist der einzige direkte Angriff auf die Grundlagen der Alchemie, der in der hier betrachteten Literatur zu finden ist. Die Kritik der anderen Autoren wendet sich stets ausschließlich gegen die Praxis.

So verhält es sich auch in BEN Jonsons Gaunerkömödie The Alchemist aus dem Jahre $1610^{34}$. Das Schauspiel stellt eine betrügerische Praxis bloß, ohne die theoretischen Grundlagen der Alchemie zu berühren.

BEn Jonson zeigt ein Gaunertrio, das sich darauf verlegt hat, aus der Dummheit und Leichtgläubigkeit der Mitmenschen Kapital zu schlagen. Es besteht aus dem betrügerischen Alchemisten Subtle, dem ungetreuen Diener Face und der zweifelhaften Dame Dol Common. Eines ihrer Gaunerkunststücke besteht in einem alchemistischen Bluff. In der Hoffnung auf große Reichtümer opfert der ebenso dumme wie gierige Sir Epicur Mam-

30 Sebastian Brandt. Das Narrenschiff, nach der Erstausgabe (Basel 1494) herausgegeben von Manfred Lemmer, 2. Auflage, Tübingen 1968, Cap. 102 "von falsch und beschiß», Zeile 49-66.

31 Ebd. Zeile 39.

32 Ebd. Zeile 68.

33 Ebd. Zeile 63-64.

34 Ben Jonson, The Alchemist, Herausgeber: Ch. M. Hathaway, New York 1903 (= Yale Studies in English, Ed. A.S. Cook. Nr.17). Zu BeN Jonson vgl. auch JoHn ReaD: The Alchemist in Life Literature and Art, London 1947, S. 39 ff. 
mon seinen ganzen Besitz für die Vollendung des großen Werkes. Natürlich mißlingt das von den flaschen Alchemisten durchgeführte Experiment. Die Apparatur fliegt in die Luft. Mammons Gold ist, zumindest für ihn, dahin. Und die drei Gauner bringen es noch fertig, ihm die Schuld an dem angeblichen Fehlschlag in die Schuhe zu schieben.

Der einzige Gegner dieser vorgeblichen Alchemie ist der skeptische Surley, ein Spieler. Der Alchemist und der Diener suchen ihn zu überzeugen. Jonson benutzt diese Diskussion zu einer ausführlichen Darstellung der alchemistischen Argumentationsweise und offenbart dabei eine beachtliche Kenntnis der hermetischen Literatur ${ }^{35}$.

Duncan hat nachgewiesen, daß die theoretischen Ausführungen der Jonsonschen Alchemisten sachlich korrekt sind und auf authentischen Vorlagen in der Fachliteratur beruhen ${ }^{36}$.

Allein durch die aufgeblasene Pose des falschen Alchemisten und des dumm-dreisten Dieners wird dieser Extrakt aus der alchemistischen Literatur zum Werkzeug der Satire.

Die musterhafte und zugleich respektlose Art, mit der Ben Jonson die ernsthafte alchemistische Philosophie zum Komödienstoff umfunktioniert, zeigt eine deutliche Distanzierung von der geheimen Wissenschaft. Andererseits weist seine profunde Kenntnis der Sache darauf hin, daß Jonson sich gründlich mit der Alchemie befaßt haben muß.

Der mittelalterliche Glaube CHAucers ist verloren gegangen, aber noch ist das Interesse an der Goldmacherkunst unübersehbar.

\section{Abschließende Überlegungen über das Bild der Alchemie in der Dichtkunst}

Die hier vorgestellten Beispiele über die Behandlung der Alchemie in der Dichtkunst entstammen einem drei Jahrhunderte umfassenden Zeitraum und sind sowohl formal wie inhaltlich sehr unterschiedlich.

Es soll jedoch abschließend versucht werden, einige gemeinsame Aspekte in dieser Literatur aufzuzeigen, die über die individuellen Aussagen der einzelnen Dichter hinausgehen. Aus diesen Gemeinsamkeiten könnte einiges über die Stellung der Alchemie im Denken der damaligen Zeit - also zwischen 1300 und 1600 - abgeleitet werden.

So ist zunächst zu bemerken, daß die Auseinandersetzung mit der Al-

35 Vgl. Jonson: The Alchemist, 2. Akt, 3. Szene, S.145-157.

36 Vgl. Edgar Hill Duncan, Jonson's Alchemist and the Literature of Alchemy, Publications of the Modern Language Association of America 61 (1946) 699-710. 
chemie für alle Autoren eine hochaktuelle Angelegenheit darstellt. Die Werke, die die Praxis oder die Theorie der Alchemie ausführlicher beschreiben, zeigen deutlich, daß die Dichter eine Sache behandeln, die sie aus eigener Anschauung kennen. Die überaus lebendigen Beschreibungen bei Petrarca und Chaucer sind kaum anders zu erklären. Und die Aussagen Chaucers und Ben Jonsons zeigen eine so genaue Kenntnis der hermetischen Literatur, daß man wohl ein persönliches Interesse an diesen Dingen vermuten darf.

Ein weiterer Aspekt, der sich aus der Betrachtung der schönen Literatur ergibt, besteht darin, daß sich bei allen Autoren eine kritische Einstellung zur Alchemie nachweisen läßt. Allein Jean de Meuns Aussage bildet hierin eine Ausnahme.

Die Kritik bezieht sich grundsätzlich auf die Praxis der Alchemie. Dabei basiert die vorgetragene Ablehnung auf der Erfahrung, daß das Ziel der Alchemie praktisch immer unerreichbar bleibt und daß scheinbare Erfolge nichts anderes sind als das Ergebnis betrügerischer Machenschaften.

Dabei fällt auf, daß niemals der ernsthafte Versuch gemacht wird, die Unmöglichkeit der Alchemie durch die Widerlegung ihrer Grundlagen zu beweisen. Während Dante und Petrarca überhaupt nicht auf die Theorie eingehen, wird die grundsätzliche Möglichkeit der Transmutation von Chaucer sogar bejaht. Sebastian Brandts Hinweis auf Aristoteles («Dann Aristoteles der gycht, die gestalt der ding wandeln sich nicht») hat eher den Charakter einer Randbemerkung. In dieser Kürze kann die alchemistische Theorie, die sich schließlich selbst in mancher Hinsicht auf Aristoteles beruft, sicher nicht hinreichend widerlegt werden.

Am ehesten könnte man bei Ben Jonson eine Außerkraftsetzung der alchemistischen Thesen erwarten, die der Betrüger Subtle so weitschweifig vorträgt. Doch der ungläubige Gegenspieler Surley verfügt über kein auch nur annähernd ernsthaftes Gegenargument.

Dies berechtigt zu der Vermutung, daß die Philosophie der Alchemie so tief im Weltbild des Mittelalters und der frühen Neuzeit verwurzelt war, daß sie nicht ohne weiteres widerlegt werden konnte. Daher mußte man allen Fehlschlägen zum Trotz zugestehen, daß das Ziel der Alchemie letztlich doch erreichbar sein könnnte. Dies aber würde etwas von der Faszination erklären, die die Königliche Kunst auf die Menschen der damaligen Zeit ausgeübt haben muß und die sie immer wieder dazu verleitet haben muß, wider alle Erfahrung, nach dem geheimnisvollen Stein der Weisen zu suchen. 